\title{
The Globulin, Albumin To Globulin Ratio(A/G) and Neutrophil To Lymphocyterate (NLR) Do Not Perform Better Than CRP and ESR in PJI Diagnosis
}

\author{
Jing-bo Jiao \\ Henan University People's Hospital \\ Jin-Cheng Huang \\ Henan Provincial People's Hospital \\ Xiao Chen \\ Henan Provincial People's Hospital \\ Yi Jin ( $\nabla$ hnjinyimd@163.com ) \\ Henan Provincial People's Hospital
}

\section{Research Article}

Keywords: globulin, albumin to globulin ratio, neutrophil to lymphocyterate, Diagnosis, Periprosthetic joint infection

Posted Date: October 26th, 2021

DOl: https://doi.org/10.21203/rs.3.rs-969948/v1

License: (9) This work is licensed under a Creative Commons Attribution 4.0 International License. Read Full License 


\title{
The globulin, Albumin to Globulin $\operatorname{ratio(A/G)~}$ and Neutrophil to Lymphocyterate (NLR) do not perform better than CRP and ESR in PJI diagnosis
}

\author{
Jing-bo Jiao ${ }^{1 \dagger}$,Jin-cheng Huang, MD, $\mathrm{PhD}^{1 \dagger}$, Xiao \\ Chen, $\mathrm{MD}^{1}$, Yi Jin, $\mathrm{MD}, \mathrm{PhD}^{1}$
}

\section{Abstract}

Objective: To test the significance of serum C-reactive protein (CRP), the erythrocyte sedimentation rate (ESR), globulin(GLB), albumin to globulin $\operatorname{ratio}(\mathrm{A} / \mathrm{G})$, and neutrophil to lymphocyterate(NLR) in periprosthetic joint infection (PJI) diagnosis.

Methods: We retrospectively analyzed the clinical data of 115 patients diagnosed from January 2017 to December 2020 with PJI (PJI group, median age 71.00 years [range, 41-94 years], 24 males, 29 females), and aseptic loosening (aseptic group, median age 68.50 years [range, 34-85 years], 32 male, 30 female) in our department. Demographic data and thesensitivity and specificity of preoperative CRP, ESR, GLB,A/G, and NLR in PJI diagnosis were compared.Results: There were no significant differences when the demographic data of the two groups were compared. The expression level of CRP (24.89 mg/L([IQR], 0.1 to 200)), ESR (3 
$\mathrm{mm} / \mathrm{h}([\mathrm{IQR}], 6$ to 120$)), \mathrm{GLB}(31.70 \mathrm{~g} / \mathrm{L}$ ( [IQR], 18.50 to 60.60$))$, and NLR (2.51([IQR], 0.93 to 12.23)) in the PJI group were higher than in the aseptic loosening group (CRP: $2.245 \mathrm{mg} / \mathrm{L}([\mathrm{IQR}], 0.2$ to 111.94);ESR: 16 mm/h ([IQR], 2 to 76$)$; GLB: $26.60 \mathrm{~g} / \mathrm{L}([\mathrm{IQR}], 17.90$ to 68.20$)$; NLR: 1.85([IQR], 0.63 to 9.09)). The expression level of $\mathrm{A} / \mathrm{G}$ (1.15([IQR], 0.55 to 2.16)) in the PJI group was lower than in the aseptic loosening group (1.51([IQR], 0.71 to 2.40)). Receiver operating characteristic (ROC) curve analysis demonstrated that the areas under the ROC curve (AUC) for CRP, ESR, GLB,A/G, and NLR were 0.841 (95\% confidence interval, 0.761$0.903), 0.850(0.771-0.910), 0.747(0.658-0.824), 0.779(0.692-0.851)$, and $0.708(0.616-0.789)$, respectively. When GLB $>26.6 \mathrm{~g} / \mathrm{L}, \mathrm{A} / \mathrm{G}<1.32$, and NLR > 2.1 were set as the threshold values for the diagnosis of PJI, The sensitivity of GLB and A/G $(90.57 \%, 81.13 \%)$ is higher than CRP $(71.70 \%)$ and ESR (79.25\%), but the specificity (GLB: $51.61 \%$, A/G: $72.58 \%$ ) was significantly lower than of CRP (87.10\%) and ESR (75.81\%). The ROC analysis of NLR showed that its sensitivity (73.58\%) and specificity (70.97) had no significant advantages over CRP and ESR.

Conclusion: globulin, A/G and NLR are not better than CRP and ESR in PJI diagnosis.

Key words: globulin; albumin to globulin ratio ; neutrophil to lymphocyterate ; Diagnosis; Periprosthetic joint infection; 
Address for correspondence Yi Jin, Department of Orthopedics, Henan Provincial People's Hospital, People's Hospital of Zhengzhou University, People's Hospital of Henan University, Zhengzhou, China, 450003. Tel: +86 17516258626, Fax: +86 13837140761; Email:

hnjinyimd@163.com (Jin)

$\dagger$ Co-first authors.

\section{Introduction}

With the improvement of average life expectancy and the emphasis on quality of life, TKA/THA has been used as a treatment for the end-stage joint diseases such as knee osteoarthritis and femoral head necrosis, TKA/THA is a effective operation to Relieve pain and enhance the quality of life. Gradually become widely accepted and more and more developed. However, accompanied by an increase in the number of operation, postoperative complications, especially the periprosthetic joint infection (PJI), have been increasingly recorded over decades ${ }^{[1-3]}$. The rate of debilitating PJI following TJA is approximately $1-2.5 \%$, produce a huge burden on the patient and health care system ${ }^{[3,4]}$.

The early clinical manifestations of PJI are not typical, and traditional serological indicators are difficult to diagnose. Therefore, finding an easily accessible blood indicator is critical for the diagnosis of PJI. Consequently, additional efforts are required to optimize PJI diagnosis by determining the 
appropriate and effective biomarkers.

In recent studies, some new blood indicators have been proposed by scholars and have shown excellent diagnostic efficacy, including FIB $^{[15]}$, globulin $^{[6]}, \mathrm{A} / \mathrm{G}$ (albumin to globulin ratio) ${ }^{[6]}$, NLR(neutrophil to lymphocyterate $)^{[5]}$. Bao-Zhan $\mathrm{YU}^{(5)}$ found that the NLR values are more accurate than CRP and may be considered as useful parameters for the diagnosis of PJI. Yongyu $\mathrm{Ye}^{(6)}$ found that Both globulin and A/G ratio were associated with PJI and may serve as potential adjuvant biomarkers in the diagnosis of PJI. Li, Rui ${ }^{[15]}$ found that FIB has good sensitivity and specificity for diagnosing PJI, with values as good as those of classical markers(CRP and ESR).

Similarly, our study ${ }^{[11]}$ also affirm the diagnostic value of FIB, but there aren't any further papers related to the diagnostic value of GLB,A/G and NLR in PJI. The exact role of GLB, A/G and NLR in PJI is still unknown. Therefore, we aimed to evaluate the diagnostic value of NLR ,Globulin and A/G ratio in PJI.

\section{Materials and Methods}

\section{Inclusion and Exclusion Criteria}

After Institutional Review Board approval for this study was obtained, we retrospectively reviewed the clinical data of patients who had been diagnosed with PJI, and aseptic loosening 
between January 2017 and December 2020.

\section{Inclusion Criteria}

The inclusion criteria were: (I) patients had been diagnosed with PJI, and aseptic loosening and received corresponding treatment (spacer insertion surgery, and revision arthroplasty) in our department from January 2017 to December 2020; (II) patients whose data were available for preoperative blood CRP, ESR, Globulin, A/G and NLR; (III) comparisons of sensitivity and specificity of preoperative CRP, ESR, Globulin ,A/G and NLR in PJI diagnosis had been made among patients from the two different groups; (IV) different expression of CRP, ESR, Globulin ,A/G and NLR among patients from the two different groups and sensitivity and specificity of CRP, ESR, Globulin , A/G and NLR in PJI diagnosis should be expected; This study design was a retrospective study.

\section{Exclusion Criteria}

The exclusion criteria include the following: patients with

(I) systemic inflammatory disease (such as rheumatoid arthritis, psoriasis, systemic lupuserythematosus, polymyalgia rheumatica, hepatitis B and C, inflammatory bowel disease, 
sarcoidosis, gout, myelodysplastic syndrome, lymphocytic leukemia, and multiple myeloma); (II) a history of recent dislocation or trauma (within 2 weeks); (III) malnutrition; (IV) tumors. ( V) missing critical data.

\section{General Information of Participants}

According to the above inclusion and exclusion criteria, the clinical data of 115 patients who had been diagnosed with PJI, and aseptic loosening from January 2017 to December 2020 were analyzed. These patients were divided into two groups according to the diagnosis: a PJI group (PJI) and an aseptic group (aseptic loosening). For each patient, demographic information (gender and age) and preoperative blood CRP, ESR, Globulin, A/G and NLR were recorded.

\section{Definition of Periprosthetic Joint Infection and Aseptic}

\section{Loosening}

Periprosthetic joint infection was defined using the MSIS

Criteria $^{[8]}$. Aseptic loosening was defined using the criteria in our previous published paper ${ }^{[7]}$. 


\section{Measuring Methods}

Preoperative blood CRP, ESR, Globulin, A/G and NLR were measured preoperatively. The sensitivity and specificity of preoperative CRP, ESR Globulin, A/G and NLR in PJI diagnosis were compared among the two different groups.

\section{Statistical Analysis}

Quantitative data were recorded as mean \pm standard deviation. $t$ test was used for comparison between two means. Nonparameter test are used when the data do not fit the normal distribution. A P-value less than 0.05 was considered significant difference. On the other hand, receiver operating characteristic (ROC) analysis was carried out to establish the diagnostic performance of blood proteins by MedCalc 19.0.4 (MedCalc Software, Ostend, Belgium). Several parameters, including sensitivity, specificity, area under the curve (AUC), and diagnostic odds ratio (DOR), were employed. Typically, AUC > 0.7 was considered acceptable. The optimal threshold for the diagnosis of PJI was determined by the Youden index. P $<0.05$ was regarded as statistically significant.

\section{Results}


There were 53 cases in infected group and 62 cases in aseptic loosening group based on the MSIS criteria. There was no significantly different in age, gender between two groups. The characteristics of each cohort are shown in (Table 1)。

Table 1: Patient characteristics

\begin{tabular}{cccc}
\hline Variable & PJI & aseptic loosening & P-value \\
\hline Sex(Male) & 24 & 32 & 0.592 \\
Sex(Femal) & 29 & 30 & \\
Age (median) & $71.0(41-94)$ & $68.5(34-85)$ & 0.214 \\
\hline
\end{tabular}

The median of Globulin was $31.70 \mathrm{~g} / \mathrm{L}$ (interquartile range [IQR], 18.50 to 60.60$)$ in the PJI group and $26.60 \mathrm{~g} / \mathrm{L}([\mathrm{IQR}], 17.90$ to 68.20) in the aseptic loosening $(\mathrm{p}<0.001)$. The median of $\mathrm{A} / \mathrm{G}$ was $1.15([\mathrm{IQR}], 0.55$ to 2.16$)$ in the PJI group and 1.51([IQR], 0.71 to 2.40 ) in the aseptic loosening group ( $\mathrm{p}<0.001)$. The median of NLR was 2.51([IQR], 0.93 to 12.23 ) in the PJI group and 1.85 ([IQR], 0.63 to 9.09) in the aseptic loosening group ( $<<$ 0.001). The median of ESR was $53 \mathrm{~mm} / \mathrm{h}$ ([IQR], 6 to 120$)$ in the PJI group and $16 \mathrm{~mm} / \mathrm{h}$ ([IQR], 2 to 76$)$ in the aseptic loosening group ( $<0.001)$. The median of CRP was $24.89 \mathrm{mg} / \mathrm{L}([\mathrm{IQR}]$, 0.1 to 200$)$ in the PJI group and $2.245 \mathrm{mg} / \mathrm{L}([\mathrm{IQR}], 0.2$ to 111.94$)$ in the aseptic loosening group $(\mathrm{p}<0.001)$. 
Table 2: Median values of diagnostic measures between PJI and aseptic loosening group

\begin{tabular}{cccc}
\hline Variable & PJI $(\mathrm{n}=53)$ & $\begin{array}{c}\text { aseptic loosening } \\
(\mathrm{n}=62)\end{array}$ & P-value \\
& & $26.60(17.90-68.20)$ & $<0.001$ \\
Globulin & $31.70(18.50-60.60)$ & $1.51(0.71-2.40)$ & $<0.001$ \\
A/G & $1.15(0.55-2.16)$ & $1.85(0.63-9.09)$ & $<0.001$ \\
NLR & $2.51(0.93-12.23)$ & $16(2-76)$ & $<0.001$ \\
ESR & $53(6-120)$ & $2.245(0.2-11.94)$ & $<0.001$ \\
CRP & $24.89(0.1-200)$ & &
\end{tabular}

In Bao-Zhan Yu's study, the ROC curve analysis of NLR showed an encouraging results, The calculated cutoff value of NLR set at 2.13, the sensitivity, specificity, was $85.0 \%, 68.3 \%$. And in Yongyu Ye's study, The calculated cutoff value of Globulin set at 29.8, and the sensitivity, specificity, was $78.95 \%, 69.66 \%$. The calculated cutoff value of $\mathrm{A} / \mathrm{G}$ set at 1.2 , and the sensitivity, specificity, was $65.79 \%, 78.65 \%$.

In this study, we use the calculated cutoff value of the references as a standard at first, the ROC curve analysis didn't show an encouraging results which has been published in the references $^{[5,6]}$. ( Table 3 )

Table3: The Sensitivity and Specificity of GLB, A/G and 
NLR in PJI diagnosis when the cutoff value was set as the references.

\begin{tabular}{ccccc}
\hline & Threshold & $(95 \% \mathrm{CL})$ & Sensitivity & Specificity \\
\hline Globulin & 29.8 & $46.0-73.5$ & 60.38 & 70.97 \\
A/G & 1.2 & $49.8-76.9$ & 64.15 & 77.42 \\
NLR & 2.13 & $55.7-81.7$ & $64.15 \%$ & $77.42 \%$ \\
\hline
\end{tabular}

Therefore, we perform an independent ROC analysis. The ROC curve analysis showed these blood parameters for diagnosing PJI, CRP (AUC =0.841) followed by the ESR (AUC $=0.850)$, NLR $(\mathrm{AUC}=0.708)$, Globulin $(\mathrm{AUC}=0.747)$ and $\mathrm{A} / \mathrm{G}$ $(\mathrm{AUC}=0.779)($ Fig. 1). We identified the cutoff values for the CRP at $14.26 \mathrm{pg} / \mathrm{ml}$. The sensitivity, specificity was 71.7 , 87.1. With the calculated cutoff value of ESR set at $32 \mathrm{~mm} / \mathrm{hr}$, the sensitivity, specificity, was 79.25,75.81. The calculated cutoff value of NLR set at 2.1, the sensitivity, specificity, was 73.58, 70.97. The calculated cutoff value of Globulin set at 26.6 $\mathrm{g} / \mathrm{L}$, the sensitivity, specificity, was $90.57,51.61$. The calculated cutoff value of $\mathrm{A} / \mathrm{G}$ set at 1.32 , the sensitivity, specificity, was $81.13,72.58$

The sensitivity of GLB and A/G $(90.57 \%, 81.13 \%)$ is higher 
than CRP (71.70\%) and ESR (79.25\%), but the specificity

(GLB: $51.61 \%, \mathrm{~A} / \mathrm{G}: 72.58 \%$ ) was significantly lower than of CRP (87.10\%) and ESR (75.81\%). The ROC analysis of NLR showed that its sensitivity (73.58\%) and specificity (70.97) had no significant advantages over CRP and ESR.

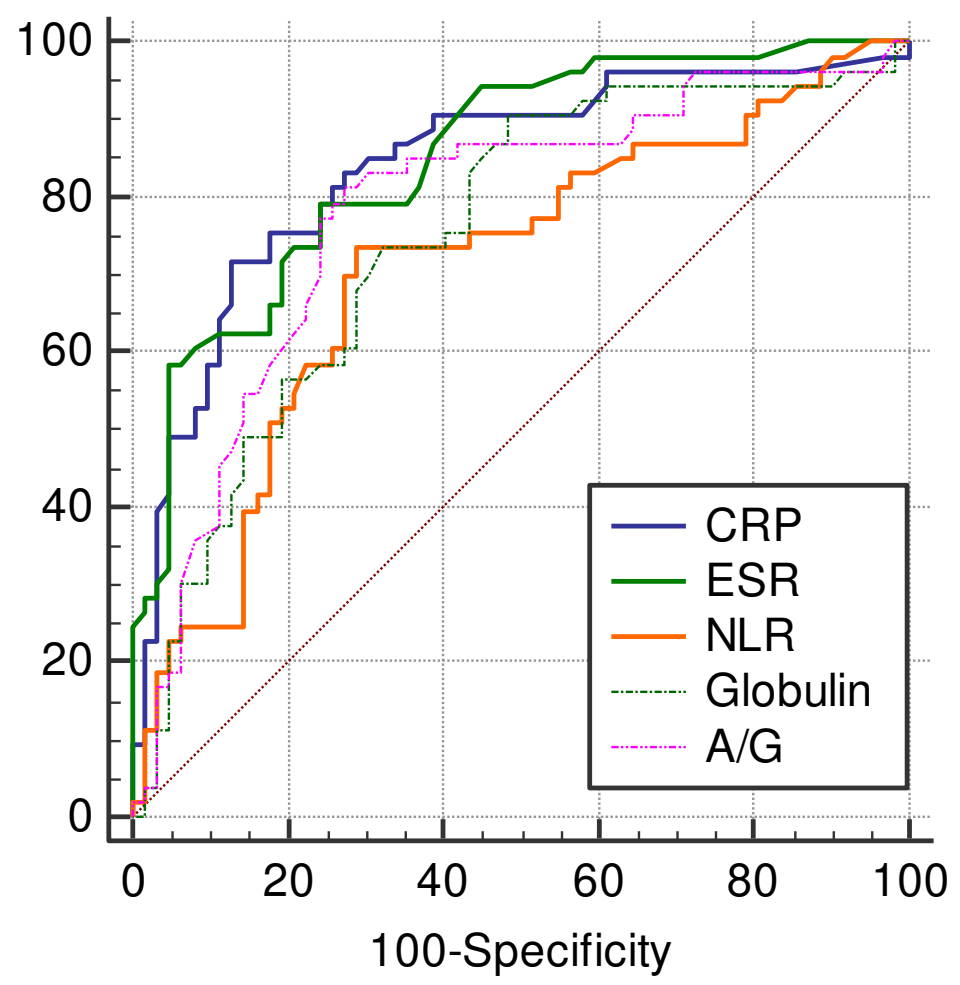

(Fig. 1:The ROC curves for C-reactive protein (CRP), erythrocyte sedimentation rate (ESR), Globulin, A/G ,neutrophil to lymphocyte ratio (NLR) in predicting PJI)

\section{Table 4: Values of blood parameters in predicting PJI}

\begin{tabular}{cccccc}
\hline & AUC & $(95 \% \mathrm{CL})$ & Threshold & Sensitivity & Specificity \\
\hline CRP & 0.841 & $0.761-0.903$ & $14.26 \mathrm{mg} / \mathrm{L}$ & 71.70 & 87.10 \\
ESR & 0.850 & $0.771-0.910$ & $32 \mathrm{~mm} / \mathrm{hr}$ & 79.25 & 75.81 \\
NLR & 0.708 & $0.616-0.789$ & 2.1 & 73.58 & 70.97 \\
Globulin & 0.747 & $0.658-0.824$ & $26.6 \mathrm{~g} / \mathrm{L}$ & 90.57 & 51.61 \\
A/G & 0.779 & $0.692-0.851$ & 1.32 & 81.13 & 72.58 \\
\hline
\end{tabular}

\section{Discussion}


The occurrence of PJI is always accompanied by the formation of bacterial biofilms $(\mathrm{BBF})$ on the surface of the implant, after the bacterias adhere to the surface of the implant, it produce a hydrated matrix of extracellular polysaccharides (glycocalyx) and protein, forming a slimy layer defined as biofilm ${ }^{(10)}$. These biofilms provide a barrier that reduces penetration of antimicrobial agents ${ }^{(9)}$. Once the $\mathrm{BBF}$ has been formed, the antibiotics can just eliminate the planktonic bacteria, after our treatment of antibiotics, the symptoms transiently disappear ,but the BBF still remain and release bacteria, resulting in another infection ${ }^{(10)}$. The existence of bacterial biofilms brings difficulties to the diagnosis of PJI, especially in patients with chronic PJI. Clinically, compared with acute PJI patients, chronic PJI patients tend to have insidious onset and atypical symptoms, which are more difficult to diagnose. Therefore, many clinicians have tried a variety of methods to diagnose PJI, the most common of which is joint puncture and bacterial culture of specimens. However, due to the existence of bacterial biofilms, joint puncture can not get satisfactory and accurate results sometimes. At the same time, joint cavity puncture also carries the risk of infection, which may induce or even cause the 
occurrence or aggravation of joint cavity infection. Therefore, some new techniques are trying to apply to the diagnosis of PJI, such as matrix-assisted laser desorption/ionization time of flight mass spectrometry,MALDI- TOF MS, PCR ， 16S rRNA, metagenomic next-generation sequencing, mNGS, But published studies can't prove that these techniques have a great value in the diagnosis of PJI.In addition, due to the limitation of high expense, these new technologies cannot be widely used in clinical practice.But in contrast, the traditional blood test still remains the preferred diagnostic index for PJI. In the past a few years, the value of numerous blood markers, such as serum soluble intercellular adhesion molecule-1 (sICAM-1), myeloid-related protein14 (MRP-14), soluble urokinase plasminogen activation receptor (su-PAR), and lipopolysaccharide-binding protein(LBP), has been tested in PJI diagnosis. Although these markers have shown good performance in PJI diagnosis, due to high expense and the existence of special antibodies, it is often not possible to access them in clinical practice ${ }^{(11)}$. We still need to find a convenient and efficient blood tests for PJI diagnosis.

WBC, CRP and ESR are classic markers and commonly used to diagnose infectious diseases, but they can't show a sufficient 
sensitivity and specificity in the diagnosis of PJI. Bedair et al.[4] reported that the sensitivity of CRP in serum for diagnosing early PJI was only $53 \%$.

Therefore, it has become a new direction for researchers to find the relationships between the PJI and common blood tests. And someone has been recognized. Alisina's ${ }^{[12]}$ study shows that serum D-dimer is a promising marker for the diagnosis of PJI. This test may also have a great utility for determining the optimal timing of reimplantation. D-dimer have also been included in the diagnostic guidelines of $\mathrm{PJI}^{[13]}$. Eventhough, there still have a study shows that The serum D-dimer does not take any advantage over the CRP and ESR for diagnosis of $\mathrm{PJI}^{[14]}$.

In earlier studies, Angkananard T's study shows that the NLR could been used as an available indicator to predict outcomes of infected patients. ${ }^{[16]}$

Meyer $\mathrm{E}^{[17]}$ found that the $\mathrm{A} / \mathrm{G}$ ratio can represent the infection in our body .Moreover, Schmilovitz-Weiss $\mathrm{H}^{[18]}$ found that the $\mathrm{A} / \mathrm{G}$ ratio is a efficient marker to predict outcomes in cancer patients.

Based on these results ,many researchers were attracted to find the association between the NLR, Globulin ,A/G and PJI. 
Bao-zhan YU's study found that NLR values are more accurate than CRP and may be considered as useful parameters for the diagnosis of early PJI. But this study showed that NLR could be used for the diagnosis of PJI, but the diagnostic efficacy was lower than CRP and ESR. The probable reasons are analyzed as follows: 1、Bao-zhan YU's study mainly included patients with early PJI, while this study mainly included patients with chronic PJI, cause the majority of clinically confirmed patients with chronic PJI were found .Therefore, The conclusion of this study may have more clinical guiding significance.

Yongyu Ye's research shows both globulin and A/G ratio were associated with PJI and may serve as potential adjuvant biomarkers in the diagnosis of PJI. But He did not compare the diagnostic value of the globulin and $\mathrm{A} / \mathrm{G}$ ratio with $\mathrm{CRP}$ and ESR. In this study, the results also shows that Globulin and $A / G$ could be used in the diagnosis of PJI, but their diagnostic efficacy didn't better than the CRP and ESR.

\section{Limitations:}

(1) the number of included patients in our study is only 149;

(2) we excluded patients with acute PJI, which account for a small percentage of patients in our department; 


\section{Conclusions:}

Our study reveals that globulin, A/G and NLR do not perform better than CRP and ESR in PJI diagnosis. However, a largescale study is still needed to finally affirm the diagnostic value of GLB,A/G and NLR in PJI.

\section{Abbreviations:}

(I) C-reactive protein (CRP),

(II) the erythrocyte sedimentation rate (ESR),

(III) globulin(GLB),

(IV) albumin to globulin $\operatorname{ratio}(\mathrm{A} / \mathrm{G})$,

(V) neutrophil to lymphocyterate(NLR)

(VI) periprosthetic joint infection (PJI)

\section{Declaration:}

\section{(I) Ethics approval and consent to participate}

This study was conducted in accordance with the Declaration of Helsinki (Ethical Principles for Medical Research Involving Human Subjects) and was approved by the ethics board of Henan Provincial People's Hospital.

\section{(II) Consent for publication}

Not applicable 


\section{(III) Availability of data and materials}

The datasets used or analysed during the current study are available from the corresponding author on reasonable request.

\section{(IV) Competing interests}

The authors declare that they have no competing interests.

\section{(V): Funding}

This study was supported by the Key Scientific and Technological Projects in Henan Province(202102310113) and the National Natural Science Foundation of China (82002840 and 82002300).

\section{(VI) Authors' contributions:}

Jing-bo Jiao performed the data analyses and wrote the manuscript.

Jin-cheng Huang, contributed significantly to analysis.

Xiao Chen contributed significantly to manuscript preparation.

Yi Jin contributed to the conception of the study.

The authors read and approved the final manuscript.

\section{（VII）Acknowledgements}

Not applicable.

\section{(VIII) Authors' information}

Department of Orthopaedics, Henan University People's Hospital and Department of Orthopaedics, Henan Provincial 


\section{People's Hospital ,Zhengzhou, China}

\section{References:}

1. Liu JZ, Saleh A, Klika AK, Barsoum WK, Higuera CA. Serum inflammatory markers for periprosthetic knee infection in obese versus non-obese patients. J Arthroplasty. 2014;29:1880-3.

2. Parvizi J, Tan TL, Goswami K, Higuera C, Della Valle C, Chen AF, et al. The 2018 definition of periprosthetic hip and knee infection: an evidence-based and validated criteria. J Arthroplasty. 2018;33:1309-14 e2.

3. Kurtz SM, Lau E, Watson H, Schmier JK, Parvizi J. Economic burden of periprosthetic joint infection in the united states. J Arthroplasty. 2012;27:61-5 e1.

4.Huotari K, Peltola M, Jamsen E. The incidence of late prosthetic joint infections: a registry-based study of 112,708 primary hip and knee replacements. Acta Orthop. 2015;86:321-5.

5. Bao-Zhan Yu. Neutrophil to lymphocyte ratio as a predictor for diagnosis of early Periprosthetic joint infection. BMC Musculoskeletal Disorders. (2020) 21:706.

6. Yongyu Ye, Serum globulin and albumin to globulin ratio as potential diagnostic biomarkers for periprosthetic joint infection:a retrospective review. Journal of Orthopaedic Surgery and Research ,(2020) 15:459

7. Huang J, Zhang Y, Wang Z, et al. The serum level of D-dimer is not suitable for distinguishing between prosthetic joint infection and aseptic loosening. J Orthop Surg Res, 2019, 14: 407.

8. Amanatullah D, Dennis D, Oltra EG, Marcelino Gomes LS, Goodman SB, Hamlin B, et al. Hip and knee section, diagnosis, definitions: proceedings of international consensus on orthopedic infections. J Arthroplasty. 2019;34: S329-S37.

9. Gristina AG, Costerton JW. Bacterial adherence to biomaterials and tissue: the significance of its role in clinical sepsis. J Bone Joint Surg Am 1985;67:264-73.

10. Antimicrobial susceptibility of Staphylococcus aureus and Staphylococcus epidermidis biofilms isolated from infected total hip arthroplasty cases.J Orthop Sci, 2006,11 (1); 46-50. 
11. Jin-cheng Huang.Exciting Performance of Plasma Fibrinogen in Periprosthetic Joint Infection Diagnosis. Orthop Surg.2021 May ;13(3):812-816.

12. Shahi A, Kheir MM, Tarabichi M, et al. Serum D- dimer test is promising for the diagnosis of periprosthetic joint infection and timing of reimplantation $[\mathrm{J}]$. J Bone Joint Surg Am 2017, 99 (17) :1419-1427.

13. The 2018 Definition of Periprosthetic Hip and Knee Infection: An EvidenceBased and Validated Criteria. J Arthroplasty. 2018 May;33(5):1309-1314.

14. Huang J, Wang Z, Significance of serum D- dimer in the diagnosis of prosthetic joint infection, Orthopedic Journal of China, Vol.27,No.17.Sep.2019.

15. Li R, Shao HY, Hao LB, et al. Plasma fibrinogen exhibits better performance than plasma D-dimer in the diagnosis of periprosthetic joint infection. A multicenter retrospective study [J] . J BoneJoint Surg Am, 2019, 101 (7) : 613-619.

16. Angkananard T, Anothaisintawee T, McEvoy M, Attia J, Thakkinstian A.Neutrophil lymphocyte ratio and cardiovascular disease risk: a systematic review and meta-analysis. Biomed Res Int. 2018;2018:2703518-8.

17. Yoshino Y, Taguchi A, Shimizuguchi T, Nakajima Y, Takao M, Kashiyama T,et al. A low albumin to globulin ratio with a high serum globulin level is a prognostic marker for poor survival in cervical cancer patients treated with radiation based therapy. Int J Gynecol Cancer. 2019;29:1722.

18. Schmilovitz-Weiss H, Cohen M, Pappo O, Sulkes J, Braun M, Tur-Kaspa R,et al. Serum globulin levels in predicting the extent of hepatic fibrosis in patients with recurrent post-transplant hepatitis C infection. Clin Transplant.2007;21:391-7. 